\title{
Colon Capsule Endoscopy for Inflammatory Bowel Disease
}

\author{
Naoki Hosoe', Yukie Hayashi' and Haruhiko Ogata' \\ ${ }^{1}$ Center for Diagnostic and Therapeutic Endoscopy, ${ }^{2}$ Division of Gastroenterology and Hepatology, Department of Internal Medicine, Keio \\ University School of Medicine, Tokyo, Japan
}

Colon capsule endoscopy was initially introduced in 2006. Since the results of a prospective study to evaluate first-generation colon capsule endoscopy efficacy for the detection of colonic neoplasia were unsatisfactory, second-generation colon capsule endoscopy was developed and reported in 2009. In this review article, we provide an overview of the current state of colon capsule endoscopy for evaluation of inflammatory bowel disease in a clinical setting and describe our clinical experience with this technology and its application. Among the various types of inflammatory bowel diseases, ulcerative colitis is the primary target for application of colon capsule endoscopy. Nevertheless, the initial results of studies using first-generation colon capsule endoscopy to evaluate inflammation were unclear. More recently, second-generation colon capsule endoscopy has provided higher accuracy for assessment of inflammation in patients with ulcerative colitis. Colon capsule endoscopy enables noninvasive observation (with reduced volume preparation) of mucosal inflammation severity in patients with ulcerative colitis. Clin Endosc 2020;53:550-554

Key Words: Colon capsule endoscopy; Inflammatory bowel disease; Preparation

\section{INTRODUCTION}

Colon capsule endoscopy (CCE) (PillCam ${ }^{\circledR}$ Colon; Given Imaging, Ltd., Yokneam, Israel) was first introduced in 2006 by Eliakim et al. ${ }^{1}$ A prospective study with first-generation CCE (CCE-1) demonstrated that the sensitivity of CCE-1 for detecting colonic lesions was low compared to colonoscopy and the results were unsatisfactory'; thus, second-generation CCE (CCE-2) (PillCam ${ }^{\circledR}$ Colon2; Given Imaging) was subsequently developed and reported in 2009. ${ }^{3}$ The second-generation colon capsule endoscope is equipped with two high-resolution cameras providing a viewing angle of $172^{\circ}$ in front and back, senses the moving speed of the capsule endoscope, and captures 4 to 35 images per second. A prospective European

Received: August 5, 2019 Revised: November 3, 2019

Accepted: November 23, 2019

Correspondence: Naoki Hosoe

Center for Diagnostic and Therapeutic Endoscopy, Keio University School of Medicine, 35 Shinanomachi, Shinjuku, Tokyo 160-8582, Japan

Tel: +81-3-3353-1211, Fax: +81-3-3357-2778, E-mail: nhosoe@z5.keio.jp ORCID: https://orcid.org/0000-0002-4516-5648

cc This is an Open Access article distributed under the terms of the Creative Commons Attribution Non-Commercial License (http://creativecommons.org/ licenses/by-nc/3.0) which permits unrestricted non-commercial use, distribution, and reproduction in any medium, provided the original work is properly cited. multicenter study showed that the detection rate of colon polyps of $>5 \mathrm{~mm}$ using CCE-2 was almost equivalent to colonoscopy. ${ }^{4}$ In a subsequent meta-analysis, Spada et al. ${ }^{5}$ confirmed that the detection sensitivity for colonic polyps of $>6 \mathrm{~mm}$ and $>10 \mathrm{~mm}$ increased between the development of CCE-1 to CCE-2. The Spada et al. meta-analysis included 14 studies with a total of 2,420 patients (1,128 for CCE-1 and 1,292 for CCE-2). ${ }^{5}$ Results showed that CCE- 2 and CCE-1 detected $>6 \mathrm{~mm}$ polyps with $86 \%$ sensitivity ( $95 \%$ confidence interval [CI], $82 \%-89 \%$ ), and $58 \%$ sensitivity ( $95 \%$ CI, $44 \%-70 \%$ ), respectively. Polyps of $>10 \mathrm{~mm}$ were detected with $87 \%$ sensitivity (95\% CI, 81\%-91\%), and 54\% sensitivity (95\% CI, $29 \%-77 \%$ ), respectively. Currently, CCE-2 is primarily utilized in screening for colonic neoplasia, ${ }^{6}$ particularly in situations such as incomplete colonoscopy. ${ }^{7.8}$ Although CCE-2 has several limitations, including high preparation volume and cost, the demand for CCE-2 in clinical settings is gradually increasing. CCE is non-invasive and especially useful for patients with inflammatory bowel disease (IBD) and ulcerative colitis (UC) in particular. In this review article, we provide an overview of the current state of CCE-2 for evaluation of IBD in a clinical setting, and describe our clinical experience with this technology and its application. 


\section{COLON CAPSULE ENDOSCOPY FOR ULCERATIVE COLITIS}

The primary target disease for CCE among all types of IBD, is UC. Several studies evaluated CCE performance for assessment of inflammation severity in patients with UC. Sung et al. ${ }^{9}$ conducted a multicenter study investigating CCE1 utility for evaluating UC mucosal inflammation severity. The study involved 100 patients with suspected or established UC and compared colonic inflammation detected by CCE-1 and colonoscopy. The sensitivity and specificity of CCE-1 for detection of active colonic inflammation was $89 \%$ and $75 \%$, respectively. In addition, the results demonstrated that CCE-1 had a low negative predictive value (65\% [95\% CI, 43-83]) indicating that mucosal healing could not be accurately detected by CCE-1. Sung et al. ${ }^{9}$ concluded that CCE-1 was a safe procedure for monitoring mucosal inflammation associated with UC; however, they could not recommend replacing colonoscopy with CCE-1. Meister et al. ${ }^{10}$ conducted a pilot study comparing CCE-1 and conventional colonoscopy disease severity scores (modified Rachmilewitz score), and the results demonstrated a significant difference. Ye et al. ${ }^{11}$ reported a significant correlation between disease activity $(\kappa=0.751)$ and UC disease extent $(\kappa=0.522)$ when comparing CCE- 1 and colonoscopy. The discrepancy in results from the pilot studies discussed above may be related to the relatively small sample sizes of both. The first feasibility study of CCE-2 utility for assessment of mucosal inflammation severity in patients with UC disease was conducted by our research team. ${ }^{12}$ We found that the
CCE-2 procedure was completed within 8 hours in $69 \%$ of the patients and that the proportion of patients exhibiting a good or excellent cleansing level was $<50 \%$, when using a low-volume (2 L) polyethylene glycol (PEG) solution with prokinetics (mosapride citrate and metoclopramide). However, we also discovered a strong correlation between CCE-2 and conventional colonoscopy in the disease activity index (Matts endoscopic score). UC inflammation is diffuse rather than patchy, and detectable by CCE- 2 in the mucosa through gaps in feces, enabling the evaluation of mucosal inflammation severity without high cleansing efficacy. Our second study ${ }^{13}$ supported these results and demonstrated that there was substantial interobserver agreement $(\kappa=0.700)$ when assessing mucosal inflammation severity with CCE-2, even at a fair cleansing level inappropriate for the detection of colon polyps. ${ }^{14}$ A previous study revealed higher diagnostic accuracy with CCE-2, versus colonoscopy for evaluating disease activity in pediatric UC patients. ${ }^{15}$ Shi et al. ${ }^{16}$ conducted a prospective study to evaluate the CCE-2 effectivity for detection of mucosal lesions and disease activity in patients with UC. The study included 150 patients with UC, and showed that CCE-2 accurately detected mucosal lesions and disease activity. It is important to note that the estimated severity of endoscopic disease calculated by CCE- 2 may be overestimated compared to colonoscopy, since the colonic lumen is not fully inflated during the examination. However, Shi et al. ${ }^{16}$ and our data ${ }^{12}$ revealed a strong correlation between the inflammation scores determined by CCE and colonoscopy, dispelling any concerns. Furthermore, using statistical methods we developed a score
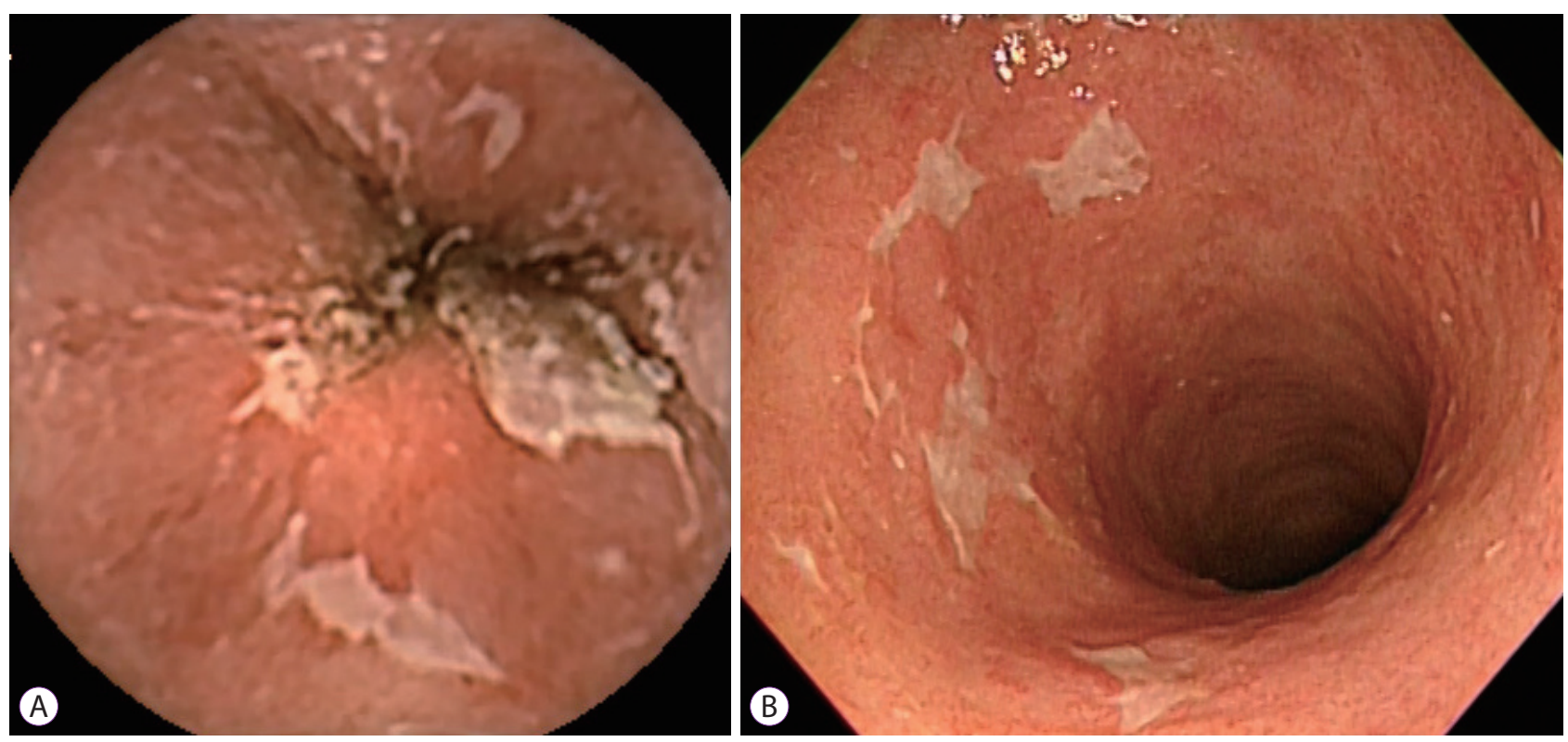

Fig. 1. (A) Colon capsule image of ulcers in a patient with ulcerative colitis (sigmoid colon). (B) Conventional colonoscopy image of ulcers in a patient with ulcerative colitis (sigmoid colon). 
specifically for CCE-2, i.e., the Capsule Scoring of Ulcerative Colitis (CSUC). The CSUC score consists of three simple items and can be easily generated using capsule endoscopy software. The correlation between the CSUC and clinical scores, such as fecal calprotectin, was almost equivalent to the Ulcerative Colitis Endoscopic Index of Severity. ${ }^{17}$ The CCE performance in assessment of UC disease extent was reported by Ye et al., ${ }^{11}$ and the study results demonstrated that there was a significant correlation between CCE- 1 and colonoscopy $(\kappa=0.522$, $p<0.001$ ). We investigated the correlation between CCE-1 and colonoscopy for detection of UC disease severity in each segment (cecum, ascending colon, transverse colon, proximal left-sided colon, and distal left-sided colon) and found strong correlations, with Rho values of $0.8620 .906,0.778,0.765$, and 0.673 for each section, respectively. ${ }^{12}$ However, further validation will be needed to determine whether CCE- 2 can accurately determine the extent of UC inflammation.

In summary, the application of CCE-1 to evaluate inflammation produced uncertain results. However, the accuracy of inflammation assessment in patients with UC has been improved by the development of CCE-2. These improvements may result from new technology increasing the capsule frame rate from 4 to 35 images per second, allowing adequate imaging of the mucosa when the capsule is accelerated by peristalsis. At present, CCE-2 has the potential to become an inflammation monitoring technique for UC, however, it is not applicable for detection of colitis associated with cancer. Large-scale studies are needed to confirm the clinical utility of CCE-2 for UC.

The typical characteristics of UC-associated inflammation are shown in Fig. 1. CCE-2 (Fig. 1A) and conventional colonoscopy (Fig. 1B) identify almost identical ulcers in the sigmoid colon.

\section{PREPARATION OF COLON CAPSULE ENDOSCOPY FOR ULCERATIVE COLITIS}

Previous studies showed that preparations including large volumes of laxatives reduce UC patient acceptance of colonoscopy ${ }^{18}$ Moreover, our clinical experience indicates that when the severity of inflammation is evaluated by CCE-2, a high cleansing level is not mandatory. The inflammation associated with UC is not patchy, but rather diffuse and continuous; thus, it is detectable in the mucosa through gaps in residual feces allowing estimation of the mucosal inflammation extent. We evaluated the feasibility of a reduced bowel preparation regimen for UC in a previous study. ${ }^{12}$ As discussed above, the

Table 1. Characteristics of Preparation and Performance of Colon Capsule Endoscopy for Ulcerative Colitis

\begin{tabular}{|c|c|c|c|c|c|c|c|c|c|}
\hline Study & $\begin{array}{c}\text { Number of } \\
\text { analyzed } \\
\text { patients }\end{array}$ & $\begin{array}{c}\text { Use of } \\
\text { purgative }\end{array}$ & $\begin{array}{l}\text { Total } \\
\text { liquid } \\
\text { (L) }\end{array}$ & $\begin{array}{c}\text { Use of } \\
\text { prokinetics }\end{array}$ & $\begin{array}{c}\text { Total colon } \\
\text { observation } \\
(\%)\end{array}$ & $\begin{array}{l}\text { Used type } \\
\text { of CCE }\end{array}$ & $\begin{array}{c}\text { Sensitivity } \\
\text { (\%) }\end{array}$ & $\begin{array}{c}\text { Specificity } \\
(\%)\end{array}$ & Correlation \\
\hline $\begin{array}{l}\text { Sung et al. } \\
(2012)^{9}\end{array}$ & 96 & PEG, NaP & 4.0 & Metoclopramide & 83 & CCE-1 & 89 & 75 & N/A \\
\hline $\begin{array}{l}\text { Meister et al. } \\
(2013)^{10}\end{array}$ & 13 & PEG-ASC & 4.7 & Domperidone & 77 & CCE-1 & N/A & N/A & N/A \\
\hline $\begin{array}{l}\text { Ye et al. } \\
\qquad(2013)^{11}\end{array}$ & 25 & PEG, NaP & 3.8 & Itopride & $\begin{array}{l}100 \text { (using } \\
\text { EGD) }\end{array}$ & CCE-1 & N/A & N/A & $\begin{array}{c}0.751 \\
\text { (kappa) }\end{array}$ \\
\hline $\begin{array}{l}\text { Hosoe et al. } \\
(2013)^{12}\end{array}$ & 29 & PEG & 2.0 & $\begin{array}{c}\text { Mosapride, } \\
\text { metoclopramide }\end{array}$ & 69 & CCE-2 & N/A & N/A & $\begin{array}{l}0.797 \\
\text { (rho) }\end{array}$ \\
\hline $\begin{array}{l}\text { Usui et al. } \\
(2014)^{13}\end{array}$ & 20 & $\begin{array}{c}\text { PEG, } \\
\text { magnesium } \\
\text { citrate }\end{array}$ & 2.2 & $\begin{array}{c}\text { Mosapride, } \\
\text { metoclopramide }\end{array}$ & 85 & CCE-2 & N/A & N/A & N/A \\
\hline $\begin{array}{l}\text { Shi et al. } \\
(2017)^{16}\end{array}$ & 108 & PEG, NaP & 4.0 & Metoclopramide & 72 & CCE-2 & 93 & 85 & $\begin{array}{c}0.69 \\
(\mathrm{ICC})\end{array}$ \\
\hline $\begin{array}{l}\text { Okabayashi et } \\
\text { al. }(2018)^{20}\end{array}$ & 33 & $\begin{array}{l}\text { PEG-ASC, } \\
\text { castor oil }\end{array}$ & 3.0 & Metoclopramide & 94 & CCE-2 & N/A & N/A & N/A \\
\hline $\begin{array}{l}\text { Takano et al. } \\
(2018)^{19}\end{array}$ & 30 & $\begin{array}{l}\text { PEG-ASC, } \\
\text { magnesium } \\
\text { citrate }\end{array}$ & 3.4 & Mosapride & 93 & CCE-2 & N/A & N/A & N/A \\
\hline
\end{tabular}

CCE, colon capsule endoscopy; CCE-1, first-generation colon capsule endoscopy; CCE-2, second-generation colon capsule endoscopy; EGD, esophagogastroduodenoscopy; ICC, intraclass correlation coefficient; N/A, not assessed; NaP, sodium phosphate; PEG, polyethylene glycol; PEG-ASC, polyethylene glycol solution containing ascorbic acid. 
cleansing efficacy of the initial study regimen was not sufficient. Therefore, we attempted to improve the colon-cleansing efficacy and completion rate of CCE for patients with UC by adopting a modified bowel preparation. ${ }^{13}$ Patients received a maximum of $2.2 \mathrm{~L}$ of PEG and magnesium citrate solution in three divided doses. In $85 \%$ of enrolled patients, the capsule reached the rectum within battery life when a modified preparation regimen was used. In the previous study, eight different preparation regimens for UC were tested (Table 1). The standard preparation for CCE colon polyp surveillance consists of 4 to $6 \mathrm{~L}$ of laxatatives. ${ }^{2,46} \mathrm{In}$ comparison, four of the tested regimens were reduced (2.0-3.4 L) (Table 1). A PEG solution containing ascorbic acid (PEG-ASC) was recently utilized in a reduced preparation for UC. ${ }^{19,20}$ Okabayashi et $\mathrm{al}^{20}{ }^{20}$ described a reduced regimen consisting of a maximum of 3.0 L of total liquid (PEG-ASC, $2 \mathrm{~L}$; water, $1 \mathrm{~L}$ ), with a high total observation rate (94\%). Thus, it is possible to reduce the CCE preparation for patients with UC. It is clearly necessary to develop lower preparation doses, since higher laxative doses reduce patient acceptance.

\section{COLON CAPSULE ENDOSCOPY FOR CROHN'S DISEASE}

Most of the studies evaluating the efficacy of capsule endoscopy in Crohn's disease (CD) have used the small bowel capsule endoscopy. $\mathrm{CD}$ tends to involve the terminal ileum and colon, and approximately $60 \%$ of patients with established $\mathrm{CD}$ have proximal small bowel lesions. ${ }^{21,22}$ Several reports ${ }^{23-27}$ assessed the role of CCE-2 in pan-enteric examinations for CD. D'Haens et al. ${ }^{23}$ compared CCE-2 and colonoscopy to assess disease severity in 40 patients with active colonic $\mathrm{CD}$. They reported substantial agreement between CCE-2 and colonoscopy in assessing the Crohn's Disease Endoscopic Index of Severity (intraclass correlation coefficient, $0.65 ; 95 \%$ CI, $0.43-0.80$ ). Additional results from the study demonstrated high sensitivity for ulcer recognition (86\%), but low specificity (40\%). The conclusion was that CCE-2 is feasible and safe for assessment of CD activity in selected populations, however, additional studies with larger sample sizes are needed to determine its true efficacy. A recent study compared CCE2 with ileocolonoscopy, magnetic resonance enterography, and small intestine contrast ultrasonography in pediatric $\mathrm{CD}$ patients. Results demonstrated that CCE-2 was superior to the other techniques for detection of colonic $\mathrm{CD}$ lesions, with sensitivity, specificity, and positive and negative predictive values of $89 \%, 100 \%$, and $100 \%, 91 \%$, respectively. ${ }^{25}$ Small case studies also showed that CCE- 2 was safe, and played an important role in the management of patients with suspected or established $\mathrm{CD}$ who refused colonoscopy, or had incomplete examinations. ${ }^{26}$ Tjandra et al. ${ }^{27}$ compared CCE-2 and ileocolonoscopy for assessing mucosal inflammation of established CD using the Simple Endoscopic Activity Score in Crohn's Disease (SES-CD). They reported a moderate correlation for SES-CD $(0.598, p=0.004)$, and a low CCE- 2 completion rate of $61.8 \%$ within the battery life.

At present, the usefulness of CCE-2 for CD is uncertain, and verification by further large-scale studies is needed.

\section{CONCLUSIONS}

Nearly 20 years have passed since the small bowel capsule emerged, and 13 years have passed since CCE was introduced. The usefulness of CCE for colon polyp surveillance has been demonstrated in many studies, but few have focused on UC. However, CCE enables noninvasive observation of mucosal inflammation severity in UC patients and may be conducted with a reduced volume preparation. At present, the limitations of CCE- 2 are that a large volume of bowel preparation is necessary, and biopsies are not possible. In the near future, improved CCE preparation regimens including reduced total liquid volume, and increased rates of completion, will be developed for UC patients. In addition, a colon capsule endoscope equipped with the ability to perform biopsies will be engineered. As a result, application of CCE for UC patient evaluation will become widespread.

\section{Conflicts of Interest}

Naoki Hosoe conducted independent research supported by a funding contribution from Covidien. Naoki Hosoe received a research grant from Olympus. The other authors have no financial conflicts of interest.

\section{Acknowledgment}

We thank Angela Morben, DVM, ELS, from Edanz Group (www. edanzediting.com/ac), for editing a draft of this manuscript.

ORCID

Yukie Hayashi: https://orcid.org/0000-0002-3777-2261

Haruhiko Ogata: https://orcid.org/0000-0002-3304-3635

\section{REFERENCES}

1. Eliakim R, Fireman Z, Gralnek IM, et al. Evaluation of the PillCam colon capsule in the detection of colonic pathology: results of the first multicenter, prospective, comparative study. Endoscopy 2006;38:963970.

2. Van Gossum A, Munoz-Navas M, Fernandez-Urien I, et al. Capsule endoscopy versus colonoscopy for the detection of polyps and cancer. $\mathrm{N}$ Engl J Med 2009;361:264-270.

3. Eliakim R, Yassin K, Niv Y, et al. Prospective multicenter performance 
evaluation of the second-generation colon capsule compared with colonoscopy. Endoscopy 2009;41:1026-1031.

4. Spada C, Hassan C, Munoz-Navas M, et al. Second-generation colon capsule endoscopy compared with colonoscopy. Gastrointest Endosc 2011;74:581-589.e1

5. Spada C, Pasha SF, Gross SA, et al. Accuracy of first- and second-generation colon capsules in endoscopic detection of colorectal polyps: a systematic review and meta-analysis. Clin Gastroenterol Hepatol 2016;14:1533-1543.e8.

6. Rex DK, Adler SN, Aisenberg J, et al. Accuracy of capsule colonoscopy in detecting colorectal polyps in a screening population. Gastroenterology 2015;148:948-957.e2.

7. Baltes P, Bota M, Albert J, et al. PillCamColon2 after incomplete colonoscopy - a prospective multicenter study. World J Gastroenterol 2018;24:3556-3566.

8. Hussey M, Holleran G, Stack R, Moran N, Tersaruolo C, McNamara D. Same-day colon capsule endoscopy is a viable means to assess unexplored colonic segments after incomplete colonoscopy in selected patients. United European Gastroenterol J 2018;6:1556-1562.

9. Sung J, Ho KY, Chiu HM, Ching J, Travis S, Peled R. The use of Pillcam colon in assessing mucosal inflammation in ulcerative colitis: a multicenter study. Endoscopy 2012;44:754-758.

10. Meister T, Heinzow HS, Domagk D, et al. Colon capsule endoscopy versus standard colonoscopy in assessing disease activity of ulcerative colitis: a prospective trial. Tech Coloproctol 2013;17:641-646.

11. Ye CA, Gao YJ, Ge ZZ, et al. PillCam colon capsule endoscopy versus conventional colonoscopy for the detection of severity and extent of ulcerative colitis. J Dig Dis 2013;14:117-124.

12. Hosoe N, Matsuoka K, Naganuma M, et al. Applicability of second-generation colon capsule endoscope to ulcerative colitis: a clinical feasibility study. J Gastroenterol Hepatol 2013;28:1174-1179.

13. Usui S, Hosoe N, Matsuoka K, et al. Modified bowel preparation regimen for use in second-generation colon capsule endoscopy in patients with ulcerative colitis. Dig Endosc 2014;26:665-672.

14. Leighton JA, Rex DK. A grading scale to evaluate colon cleansing for the PillCam colon capsule: a reliability study. Endoscopy 2011;43:123127.

15. Oliva S, Di Nardo G, Hassan C, et al. Second-generation colon capsule endoscopy vs. colonoscopy in pediatric ulcerative colitis: a pilot study. Endoscopy 2014;46:485-492.

16. Shi HY, Chan FKL, Higashimori A, et al. A prospective study on sec- ond-generation colon capsule endoscopy to detect mucosal lesions and disease activity in ulcerative colitis (with video). Gastrointest Endosc 2017;86:1139-1146.e6.

17. Hosoe N, Nakano M, Takeuchi K, et al. Establishment of a novel scoring system for colon capsule endoscopy to assess the severity of ulcerative colitis-capsule scoring of ulcerative colitis. Inflamm Bowel Dis 2018;24:2641-2647.

18. Manes G, Fontana P, de Nucci G, Radaelli F, Hassan C, Ardizzone S. Colon cleansing for colonoscopy in patients with ulcerative colitis: efficacy and acceptability of a 2-L PEG plus bisacodyl versus 4-L PEG. Inflamm Bowel Dis 2015;21:2137-2144

19. Takano R, Osawa S, Uotani T, et al. Evaluating mucosal healing using colon capsule endoscopy predicts outcome in patients with ulcerative colitis in clinical remission. World J Clin Cases 2018;6:952-960.

20. Okabayashi S, Kobayashi T, Nakano M, et al. A simple 1-day colon capsule endoscopy procedure demonstrated to be a highly acceptable monitoring tool for ulcerative colitis. Inflamm Bowel Dis 2018;24:24042412 .

21. Mehdizadeh S, Chen GC, Barkodar L, et al. Capsule endoscopy in patients with Crohn's disease: diagnostic yield and safety. Gastrointest Endosc 2010;71:121-127.

22. Greener T, Klang E, Yablecovitch D, et al. The impact of magnetic resonance enterography and capsule endoscopy on the re-classification of disease in patients with known Crohn's disease: a prospective Israeli IBD Research Nucleus (IIRN) study. J Crohns Colitis 2016;10:525-531.

23. D'Haens G, Löwenberg M, Samaan MA, et al. Safety and feasibility of using the second-generation Pillcam colon capsule to assess active colonic Crohn's disease. Clin Gastroenterol Hepatol 2015;13:1480-1486.e3.

24. Hall B, Holleran G, McNamara D. PillCam COLON $2^{\circ}$ as a pan-enteroscopic test in Crohn's disease. World J Gastrointest Endosc 2015;7:12301232.

25. Oliva S, Cucchiara S, Civitelli F, et al. Colon capsule endoscopy compared with other modalities in the evaluation of pediatric Crohn's disease of the small bowel and colon. Gastrointest Endosc 2016;83:975-983.

26. Negreanu L, Smarandache G, Mateescu RB. Role of capsule endoscopy Pillcam colon 2 in patients with known or suspected Crohn's disease who refused colonoscopy or underwent incomplete colonoscopic exam: a case series. Tech Coloproctol 2014;18:277-283.

27. Tjandra D, Kheslat HH, Amico F, Macrae F. Colon capsule endoscopy: looking beyond the colon in Crohn's disease. Inflamm Bowel Dis 2017;23:E43-E44 